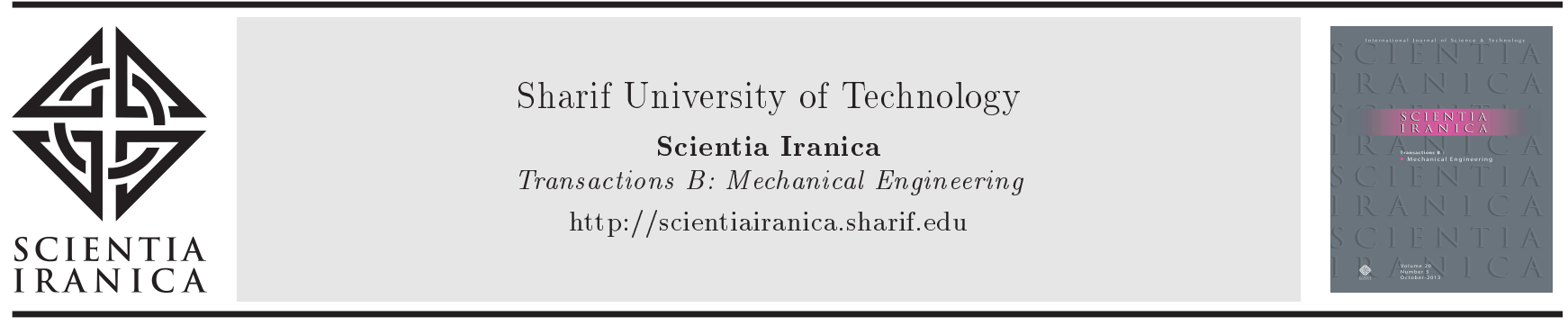

\title{
Adaptive predictive control for torque applying system of high-powered test rig
}

\author{
A. Parvaresh ${ }^{\mathrm{a}, \mathrm{b}}$ and M. Mardani ${ }^{\mathrm{b}, *}$ \\ a. Department of Mechanical Engineering, K.N. Toosi University of Technology, Tehran, Iran. \\ b. Department of Automation Engineering, Sharif Technology Branch of ACECR, Tehran, Iran. \\ Received 16 August 2020; received in revised form 8 December 2020; accepted 22 February 2021
}

\author{
KEYWORDS \\ Mechanically \\ closed-loop test rig; \\ Predictive control; \\ Torque applying \\ system; \\ Adaptive control; \\ Parameterized \\ identification.
}

\begin{abstract}
$\overline{\text { Abstract. This paper proposes the implementation of a novel predictive control scheme }}$ known as Adaptive Generalized Predictive Control (AGPC) in the actuation system of a high-powered test rig. Through the use of actuation system, the required torque for simulating different conditions can be applied to the tested gearboxes. Accurate and precise control of this system is of great importance as it affects the overall performance of the test rig. The considered actuation system in this investigation is an electro-hydraulically driven system with nonlinear and uncertain characteristics. The performance of the proposed control scheme in different conditions of the parametric uncertainty as well as the presence of disturbances is evaluated and the results are discussed. The obtained results confirmed the superior performance of the proposed scheme in different studied conditions.
\end{abstract}

(C) 2021 Sharif University of Technology. All rights reserved.

\section{Introduction}

Given the significance of evaluating high-powered gearboxes that are widely used in helicopters, test rigs are developed. By checking their overall performance through the use of test rigs, the occurrence of major failures would be minimized [1]. The test rigs are capable of providing realistic conditions which are mostly encountered during the performance with the help of changing the applied torque and speed of the system [2]. Hence, an actuation system is required for the implementation of the desired torque automatically. Among the different systems used for actuation, electro-hydraulically powered systems are of great importance given their superior characteristics. These characteristics include higher power/force generation, smaller equipment size, fast response, enhanced robust-

*. Corresponding author.

E-mail address: Mardani@acecr.ac.ir (M. Mardani) ness, and good positioning accuracy [3-5]. However, these actuators suffer the existing nonlinearities and time-dependent characteristics, which would result in backlash, friction, and increased complexity in modeling $[6,7]$. The main issue in the use of these systems for the efficient control of actuation systems is to achieve enhanced accuracy and maintain robustness.

Prior to the design of a controller, appropriate modeling of Electro Hydraulic Actuator (EHA) is very important due to its impact on the performance of the designed controller. Different approaches to modeling these actuators have been used, among which linearized mathematical models, simplified physical models, and data-driven models can be named. Considering the nonlinear and uncertain dynamics of EHA, the use of data-driven modeling is superior to other approaches [8-10]. Yao et al. [11] utilized the dynamic model of EHA in the form of mathematical relations and used a multi-layered neural network structure as a black-box estimator to overcome the mismatched and matched disturbances besides improving the compensation accuracy. In fact, the whole system was not identified and 
only the disturbances were supposed to be identified. They declared the excellent performance of the proposed strategy for estimation of existing uncertainties in the system. Lu et al. [7] used hybrid online/offline identification of the model parameters of hydraulic system including time-varying and nonlinear deformation forces. They obtained the dynamic model of the system upon considering the pressure, displacement, velocity, and acceleration data which were obtained from different experiments. The required parameters were identified using nonlinear optimization algorithm such as the particle swarm optimization method. Then, a tracking control strategy was implemented for the provision of satisfactory force and velocity for the piston rod to perform the desired task. Their modeling procedure and control scheme were validated through experimental tests. In the mentioned study, some parameters were estimated in an offline manner and were used in the subsequent online identification step, which increased the computational cost. In addition, the whole dynamics of the system was not identified.

After achieving an appropriate model for the system, the control issue can be addressed. Several investigations have been dedicated to the development of control scheme for precise position control of EHA with its usage in industrial applications. Takloo et al. [12] employed Fractional-Order ProportionalIntegral-Derivative (FOPID) controller with a mathematical model of EHA system to control the actuation system in a mechanically closed-loop test rig constructed for testing the gearboxes. They declared the improved accuracy and increased speed in comparison with conventional Proportional-IntegralDerivative (PID) controllers. In another research, Maddahi et al. [13] used the same controller for the position control of hydraulic actuators with unknown parameters. The parameters were determined through System Identification (SI) procedure. In addition, the controller parameters were tuned experimentally with an iterative algorithm to achieve tracking accuracy as well as stability. In a research study by $\mathrm{Li}$ et al. [14], a nonlinear back-stepping controller was developed for high-velocity tracking performance and achieving reduced energy consumption. In addition, RadialBased Function Neural-Network (RBFNN) was utilized to compensate the uncertainties and disturbances in the velocity control loop. In this study, the system model was obtained in the state-space form and only the load force was estimated.

The optimal control approach to achieving energy efficiency and force tracking performance was proposed by Heybroek and Sjöberg [15] for the control of EHA. This study implemented a simplified model of the system for control purposes. In addition, a Model Predictive Control (MPC) controller was utilized to achieve force control, while another controller was used for energy efficiency property. The conducted study was limited to the force control rather than motion control. Rozali et al. [16] suggested using PID controller for controlling EHA. The model of the system was obtained through SI technique by collecting the input-output data from the real experiments and implementing auto-regressive models with exogenous inputs (ARX) structure. The coefficients of PID controller were tuned by Ziegler-Nichols tuning method. Their results revealed acceptable tracking of the controller; however, the response was slow. Generally, several approaches have been proposed for the adaptive control of different systems. In [15], an output feedback control scheme was developed with the help of an unknown dynamic estimator to overcome the existing problems in previous adaptive control designs. Mostly, the traditional adaptive schemes can be classified as designs using back-stepping or dynamic surface control or the designs using intelligent approximation-based controls such as neural networks or fuzzy logic control. The former group requires direct measurement of all system states. In addition, complex implementation and stability analysis are challenging in these controllers. The latter group of controllers, despite their superior performance in the face of uncertainties and nonlinearities, suffers high computational costs derived from the tuning of several parameters. To overcome the mentioned stability issue of the prior class, a novel composite learning technique was suggested [17] which used the online recorded data together with instantaneous data to generate prediction error. In [18,19], a new Model Reference Adaptive Control (MRAC) framework with a modified reference model was proposed to improve the transient response, which was originated from possible uncertainties in the system dynamic. In addition, the stability issue was solved based on a leakage term in the adaption law to ensure the convergence.

$\mathrm{MPC}$ is an advanced and well-known control technique that is widely used for process control in many industrial applications [20]. This strategy implements the explicit model of the desired process to determine the control signal and predict the control variables over a certain time horizon and through the minimization of an objective function $[21,22]$. This controlling approach has been utilized in different applications of EHAs [2326]. In recent years, Generalized Predictive Control (GPC), which is considered as a special case of MPC with its own superiority, has gained extensive interest in various industries [27]. Through the use of this method, an equilibrium between computational cost and disturbance rejection could be ensured [28]. The problem that is mostly encountered in real applications is the presence of disturbances and model uncertainties. According to Gao [29], the term disturbance incorporates both disturbances from external environments 
and uncertainties in the un-modelled dynamics. To resolve this issue, Adaptive Predictive Control (APC) has been proposed as an efficient approach [30]. The adaptation law can be implemented by updating the parameter estimations [31]. The use of APC in different problems has yielded satisfying results $[32,33]$. Wang and Wang [34] offered the use of ultra-local MPC, which was considered a straight-forward model-free MPC procedure. The MPC was applied to the updated linear model of the system, and the requirement for the extensive dataset for training was obviated.

Upon obtaining the online model of the system through the data gathered from the experimental tests, this study used the GPC process for controlling the actuation system. The highlights of this study can be summarized as follows:

- Proposition of an efficient approach to simultaneous identification and control of a test rig with unknown parameters;

- Provision of adaptability as well as robustness to external disturbances and uncertain and changing dynamics;

- Reduction of computational cost with the help of parameterized identification with the least unknown parameters and predetermination of the system structure;

- The use of parameterized control sequence calculations by piece-wise linear functions that pass the defined points over a time horizon to reduce the controller computational cost;

- Achievement of high-accuracy tracking performance in unstable conditions.

The rest of the paper is organized as follows. in Section 2, the proposed approach to the identification and control of the electro-hydraulically driven actuation system is detailed. Section 3 is dedicated to the demonstration of experimental setup which is supposed to be controlled. After that, the obtained results are presented and discussed in Section 4. Finally, the chief findings of this study are provided in the conclusion section.

\section{Problem definition}

In this study, Adaptive Generalized Predictive Control (AGPC) is used for controlling the actuation system. As mentioned, automatic tuning of controller parameters is of great significance in industrial applications. In this scheme, updating the model parameters and regulating the coefficients are performed by an automatic tuner [35]. The optimization problem is solved at each step considering the system model and current information to find the optimum control signal. Then, the first element of the obtained signal is applied to the system and the mentioned optimization problem is solved again using the new information [27]. The procedure is defined as in the following.

Consider an unknown system whose function may change over time and that only the input-output data are available for this system. The studied system in this paper is a Single-Input Single-Output (SISO) system that can be described by a Controller Auto Regression Integrated Moving Average (CARIMA) model as follows:

$$
A\left(z^{-1}\right) y(k)=B\left(z^{-1}\right) u(k)+C\left(z^{-1}\right) \frac{\xi(k)}{\Delta},
$$

where $y(k), u(k) \in \mathbb{R}$ represent the output and input of the system, respectively. The integer $k=0,1, \ldots$ is the discrete time sample and $z^{-1}$ denotes the onestep backward operator; therefore, we define $z^{-i} x(k)=$ $x(k-i)$ and $z^{-i} u(k)=u(k-i)$. In addition, $\xi(k)$ is an uncorrelated random noise with zero mean which is added to the model by the operator $\Delta=1-z^{-1}$, which is a difference operator. In Eq. (1), $A\left(z^{-1}\right), B\left(z^{-1}\right)$, and $C\left(z^{-1}\right)$ are the polynomials with the respective degrees of $n a, n b$, and $n c$ defined as:

$$
\begin{aligned}
& A\left(z^{-1}\right)=a_{n a} z^{-n a}+\ldots+a_{1} z^{-1}+1=1+\sum_{1}^{n a} a_{i} z^{-i} \\
& B\left(z^{-1}\right)=b_{n b} z^{-n b}+\ldots+b_{1} z^{-1}+b_{0}=\sum_{1}^{n b} b_{i} z^{-i} \\
& C\left(z^{-1}\right)=c_{n c} z^{-n c}+\ldots+c_{1} z^{-1}+c_{0}=\sum_{1}^{n c} c_{i} z^{-i}
\end{aligned}
$$

For the sake of simplicity, if white noise is considered, $C\left(z^{-1}\right)=1$. Thus, we have:

$$
A\left(z^{-1}\right) y(k)=B\left(z^{-1}\right) u(k)+\frac{\xi(k)}{\Delta} .
$$

Here, Diophantine equation is used for deriving the past and future dynamics, which can be used for prediction purposes. In order to predict output $y(k+j \mid k)$ at time step $k+j$ based on available information at time $k$, Diophantine equation is introduced as follows:

$$
\begin{aligned}
& 1=E_{j}\left(z^{-1}\right) \Delta A\left(z^{-1}\right)+z^{-j} F_{j}\left(z^{-1}\right) \\
& \left\{\begin{array}{l}
E_{j}\left(z^{-1}\right)=\sum_{i=0}^{j-1} e_{j, i} z^{-1} \\
F_{j}\left(z^{-1}\right)=\sum_{i=0}^{j-1} f_{j, i} z^{-1}
\end{array}\right.
\end{aligned}
$$

In the above equation, $E_{j}\left(z^{-1}\right)$ and $F_{j}\left(z^{-1}\right)$ are the polynomial expressions. By the use of the abovementioned definitions and some simplifications, $y(k+j \mid k)$ can be obtained as follows:

$$
\begin{aligned}
y(k+j \mid k)= & E_{j}\left(z^{-1}\right) B\left(z^{-1}\right) \Delta u(k+j-1 \mid k) \\
& +F_{j}\left(z^{-1}\right) y(k) .
\end{aligned}
$$

The optimization problem is defined by considering a quadratic cost function as: 


$$
\begin{aligned}
J= & \sum_{j=1}^{P}\left(y(k+j \mid k)-W_{\text {des }}(k+j \mid k)\right)^{2} \\
& +\sum_{j=1}^{V} \lambda(j)(\Delta u(k+j-1 \mid k))^{2},
\end{aligned}
$$

where $P$ and $V$ are the prediction and control horizons, respectively, $W_{\text {des }}$ represents the desired reference for the system, and $\lambda(j)$ is the control weighting coefficient, which is considered as a constant value for simplicity. An attempt is made to minimize the $J$ index subjected to existing constraints; therefore, we have:

$$
\frac{\partial J(t)}{\partial u(t)}=0 .
$$

The control signal increment can be obtained from:

$$
\Delta U=\left(G^{T} G+\lambda I\right)^{-1} G^{T}(W-f) .
$$

In the above equation, $f$ is a vector which consists of past inputs, past outputs, and current output. In addition, $G$ denotes the step response elements. The matrices $G$ and $f$ are defined as follows:

$$
\begin{aligned}
G & =\left[\begin{array}{cccc}
g_{p} & g_{p-1} & \cdots & g_{p-v+1} \\
g_{p+1} & g_{p} & \cdots & g_{p-v+2} \\
\vdots & \vdots & \ddots & \vdots \\
g_{v} & g_{v-1} & \cdots & g_{v-p+1}
\end{array}\right], g_{j}=0, \forall j \leq 0 \\
f & =\left[\begin{array}{llll}
f_{1}(k) & f_{1}(k) & \ldots & f_{P}(k)
\end{array}\right]
\end{aligned}
$$

According to the receding horizon theory, only the first element of the obtained sequence is applied to the plant, $u(t)=u(t-1)+\Delta u(1)$, but the equation is solved over the entire horizon of time. This would lead to increased computational cost, which may not be fast enough to be implemented in real-time control applications, especially in the case of long-term horizon or high inputs. To this end, it is proposed that the dimensions of the search space be reduced by parameterizing the control sequence [36]. The idea is analogous to the curve fitting so that instead of using many discrete points, the sequence is parameterized by piece-wise linear functions that pass the defined points. With the use of this procedure, the implementation time is shortened significantly.

The possible uncertainties in the plant dynamic as well as the external or internal disturbances may affect the plant representation, i.e., the polynomials of $A\left(z^{-1}\right)$ and $B\left(z^{-1}\right)$. The explanation represented in Eq. (3) can be rewritten as follows:

$$
A\left(z^{-1}\right) \Delta y(k)=B\left(z^{-1}\right) \Delta u(k)+\xi(k) .
$$

In the above equation, we have some known values including $\Delta y(k)$ and $\Delta u(k)$ and unknown values including coefficients of $A\left(z^{-1}\right)$ and $B\left(z^{-1}\right)$ polynomials.
The vectors of unknown parameters $(\theta)$ and the vectors of known values $(\phi(k))$ are:

$$
\begin{aligned}
& \theta=\left[\begin{array}{llllll}
a_{1} & \ldots & a_{n a} & b_{1} & \ldots & b_{n b}
\end{array}\right], \\
& \phi(k)=\left[\begin{array}{ccc}
\Delta y(k-1) & \ldots & \Delta y(k-n a) \\
\Delta u(k-1) & \ldots & \Delta u(k-n b)
\end{array}\right] .
\end{aligned}
$$

Regarding the above-mentioned definitions, Eq. (10) can be written as follows:

$$
\Delta y(k)=\phi^{T}(k) \theta+\zeta(k) .
$$

Hence, in order to adapt to the new conditions, parameters should be identified and updated online. The identification procedure can be applied through different procedures. Recursive Least Square (RLS) estimation is among the most common procedures proposed for online identification of the plants. The control scheme, which requires online adjustment of system parameters, is often called adaptive control. Another point to note is the order of the considered model. The order of the system can be chosen based on the evaluation of performance besides computational cost of the different systems. This requires a multi-objective optimization, which increases the computational cost of the whole procedure if it is done in each iteration. In this research, the selection of the model was performed on the basis of Parsimony principle. This principle states the priority of selecting a model with fewer parameters and acceptable accuracy among the models with different orders [37]. The selection of a model with fewer parameters would lead to lower computational cost as well as a simpler design of the controller [38].

Accordingly, the order of the system is defined prior to the initiation of adaptive identification process and it is considered as a known parameter in the algorithm. The proposed algorithm is represented in Figure 1. The estimation of the parameter for the time step $k$, which is represented by $\widehat{\theta}(k)$, is defined by the following algorithm, which is known as RLS algorithm. For more detail about this algorithm, refer to [39]:

$$
\begin{aligned}
\widehat{\theta}(k)= & \theta(k+1)+L(k)\left[y(k)-\phi^{T}(k) \theta(k-1)\right], \\
L(k)= & \frac{p(k-1) \phi(k)}{\lambda(k)+\phi^{T}(k) p(k-1) \phi(k)}, \\
p(k)= & \frac{1}{\lambda(k)} \\
& {\left[p(k-1)-\frac{p(k-1) \phi(k) \phi^{T}(k) p(k-1)}{\lambda(k)+\phi^{T}(k) p(k-1) \phi(k)}\right] . }
\end{aligned}
$$

\section{Experimental set-up}

In this paper, the torque applying system, which is an electro-hydraulically-driven system, is investigated. 


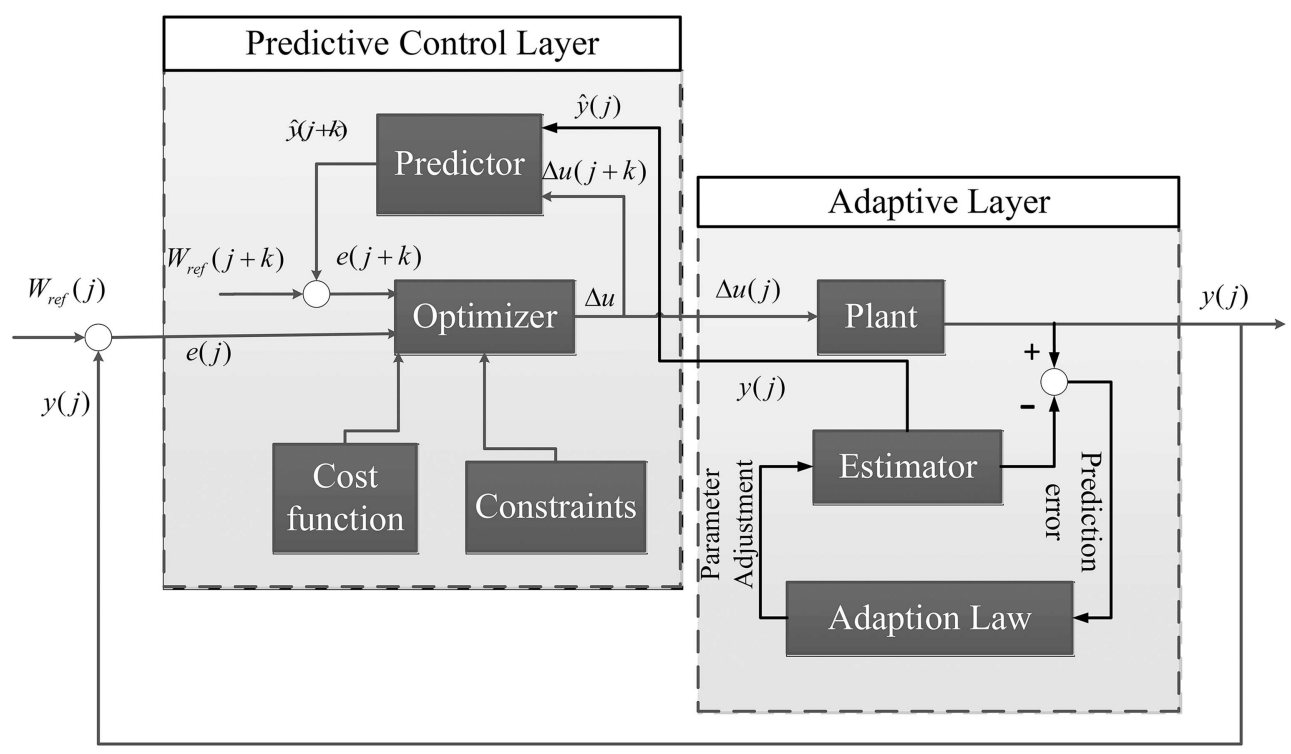

Figure 1. The proposed Adaptive Generalized Predictive Control (AGPC) algorithm for the control of Electro Hydraulic Actuator (EHA) in the actuation system of the high-powered test rig.
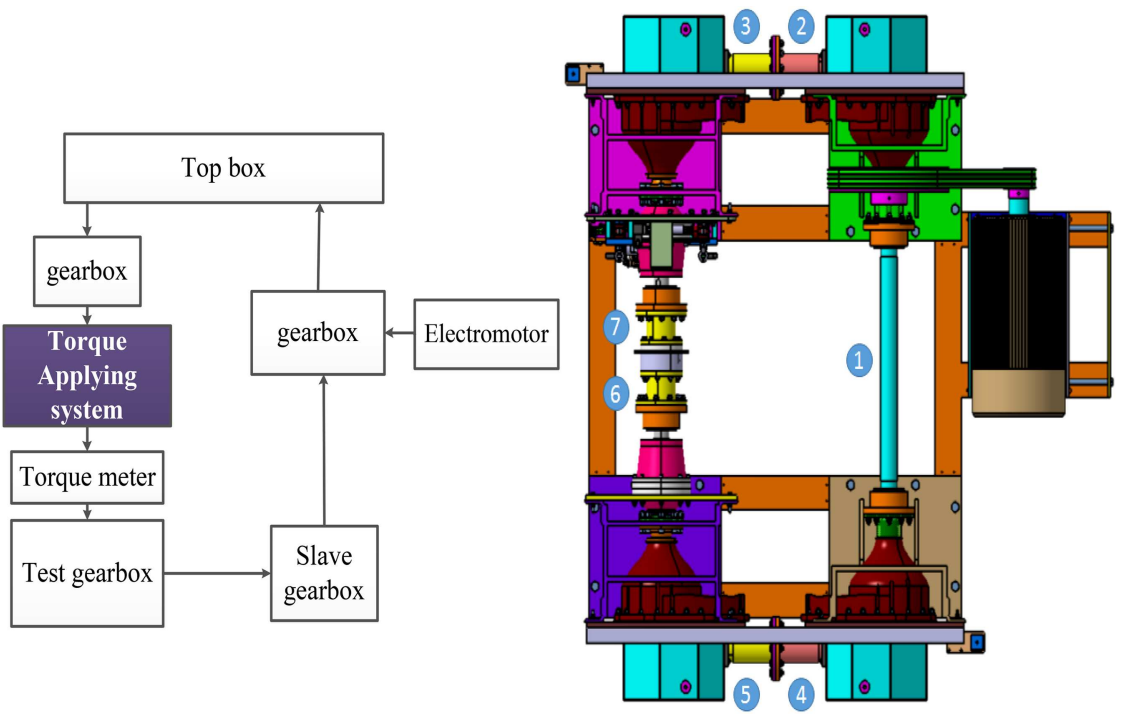

Figure 2. Schematic of the designed test rig for the high-powered test rigs.

This torque applying system is used in a closed-loop test rig, which is designed for testing high-powered gearboxes of helicopters. The mentioned test rig was designed and fabricated in Sharif University of Technology, Branch of Academic Centre of Education, Culture and Research (ACECR). Low energy loss and provision of various torques and speeds are among the superior characteristics of the mentioned test rig. According to Figure 2, the energy in this system circulates in a closed-loop manner and the embedded motor is used for the compensation of energy loss as well as provision of initial power for the system start. A view of the fabricated test rig is depicted in Figure 3. To apply the required torque in the test rig to simulate different operational conditions, EHA along with planetary gearboxes is used. The linear motion of EHA is applied to the pins, as depicted in Figure 4, in which the motion is transmitted to the rotation of the ring and the required torque is generated for the rotation of testing components. Hence, the evaluation of performance besides fault detection could be possible by studying the system under different conditions. The characteristics of the system are provided in Table 1.

\section{Results and discussions}

\subsection{Obtaining the order of the model}

In order to reduce the computational cost, the order of the model should be identified prior to the control scheme. Therefore, the model order would be a given 
Table 1. The characteristics of the test rig and the torque applying system.

\begin{tabular}{lll}
\hline & \multicolumn{1}{c}{ Specification } & \multicolumn{1}{c}{ Value } \\
\hline \multirow{3}{*}{ Test rig } & Type & Mechanically closed loop \\
& Max loading capacity & $489 \mathrm{HP}$ \\
& Max rotational speed & $3000 \mathrm{rpm}$ \\
& & \\
& Type & Hydraulically driven \\
& Max hydraulic actuator course & $60 \mathrm{~mm}$ \\
Torque applying system & Max required force & $10 \mathrm{KN}$ \\
& Force applying arm & $175 \mathrm{~mm}$ \\
& Servo valve & MOOG G763004 \\
& Max rotation & $20 \mathrm{deg}$ \\
& & \\
Sensor & Displacement sensor & Opkon Lpc-LM 75 \\
\hline
\end{tabular}

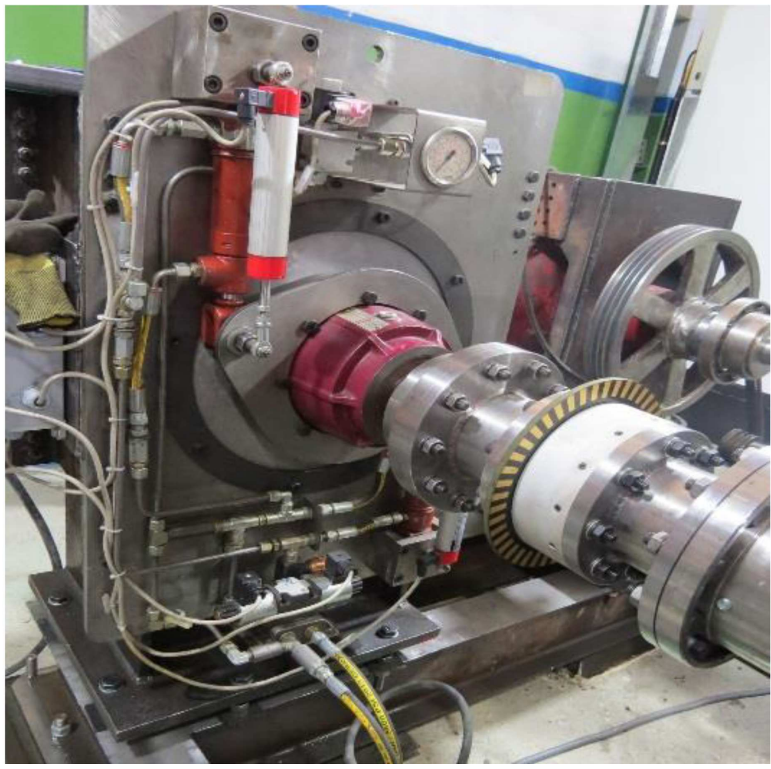

Figure 3. A view of the fabricated test rig for the high-powered gearboxes at Academic Centre of Education, Culture and Research (ACECR).

parameter in AGPC algorithm, while other parameters should be identified using the algorithm. To determine the appropriate order of the model, the linearized models with different orders were tested and compared in terms of accuracy and computational cost. In order to collect the required data for modeling, the input signals (voltage to the EHA) were applied to the hydraulic actuation system and the resultant outputs (displacement of EHA rod) were measured by the MOOG G763004 sensor. The applied excitation signal is illustrated as follows:

$$
S_{\text {exc }}=\sum_{i=1}^{l} s_{i} \cos \omega_{i} t_{s}=s_{1} \cos \omega_{1} t_{s}+s_{2} \cos \omega_{2} t_{s}+\cdots
$$

In the above equation, $S_{\text {exc }}$ represents the input signal, $l$ is the number of sine signals to be combined, and $t_{s}$

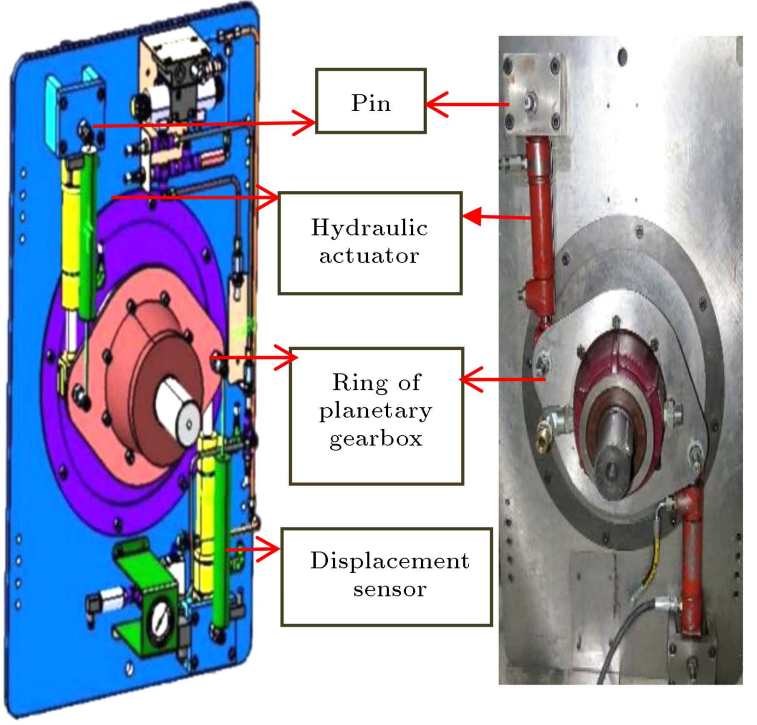

Figure 4. Real status and schematic of the torque applying system for actuation of the high-powered test rig.

denotes the sampling time. Moreover, the amplitude and frequency of the excitation signal are represented by $s_{i}$ and $\omega_{i}$, respectively. The implemented system for gathering the data is depicted in Figure 5, while the input-output data measured from real experiments on EHA are represented in Figure 6. In the following, the modeling of the plant is discussed.

In this section, structures with different orders are used to identify the system. The relationship between the inputs and outputs of the system for $n$ order structure can be defined as follows:

$$
y(t)=\frac{a_{1 x} z^{-1}+a_{2 x} z^{-2}+\ldots+a_{n x} z^{-n}}{1-b_{1 x} z^{-1}-b_{2 x} z^{-2}-\ldots-b_{n x} z^{-n}} u(t),
$$

where $y(t)$ and $u(t)$ are the outputs and inputs of the system, respectively, which are collected from the measurement sensors. In addition, $z^{-n}$ represents the order of the system. Moreover, $a_{i x}$ and $b_{i x}$ are the 


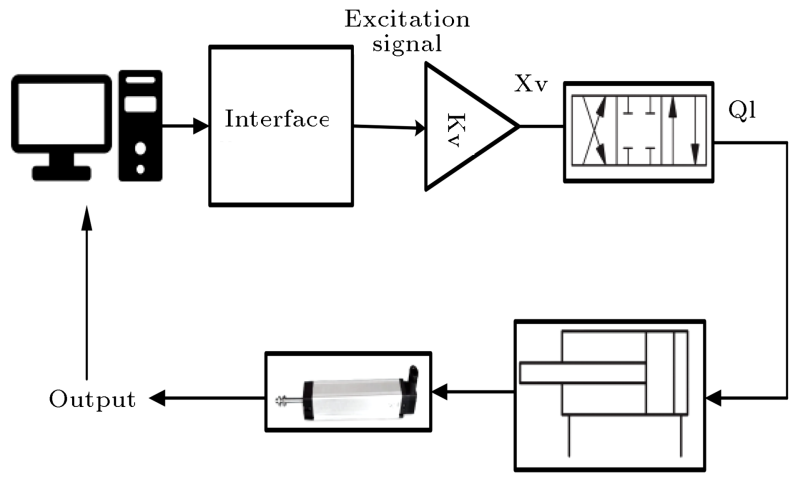

Figure 5. Data-acquisition procedure for gathering the data of the torque applying system.

unknown parameters that should be identified. The structure can be defined as follows:

$$
\widehat{y}(t \mid \theta)=\phi^{T}(t) \cdot \theta \text {. }
$$

In this definition, $\widehat{y}(t \mid \theta)$ is the estimated value; $\phi^{T}(t)$ and $\theta$ are the regressor vectors and unknown parameters vectors, respectively, which are defined as follows:

$$
\begin{aligned}
& \theta=\left[\begin{array}{llllllll}
a_{1 x} & a_{2 x} & \cdots & a_{n x} & b_{1 x} & b_{2 x} & \cdots & b_{n x}
\end{array}\right], \\
& \phi(t)=[y(t-1) \cdots y(t-k) u(t-1) \cdots u(t-k)],
\end{aligned}
$$

This paper investigated first-order, second-order, and third-order structures. According to the results of
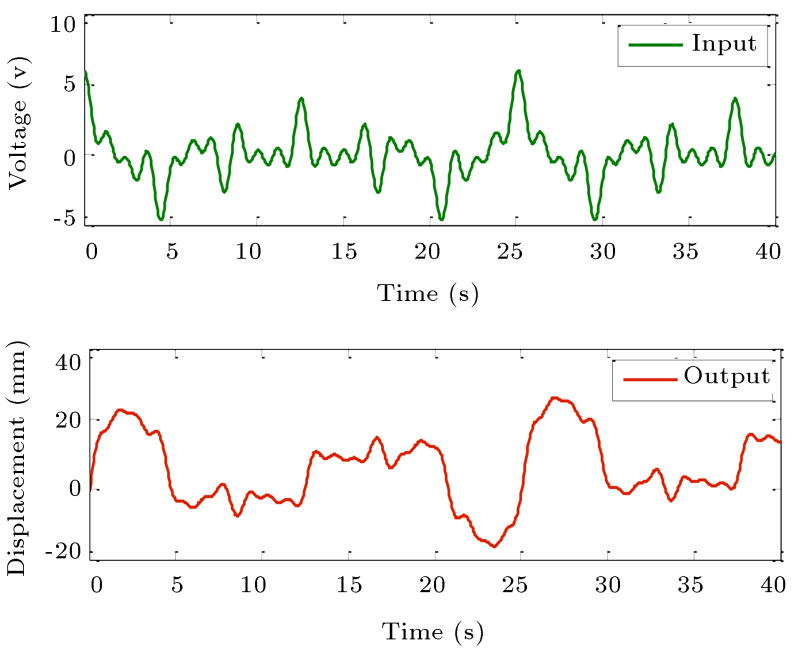

Figure 6. The gathered input-output data from the excitation of the torque applying system.

Root Mean Square Error (RMSE) for different orders of the model provided in Table 2 and outputs of the system represented in Figure 7, it can be inferred that the third-order structure provides the best results. However, regarding the computational cost as another influential parameter, the second-order structure was selected. In other words, it can be stated that the model order was selected so that an acceptable compromise between the accuracy and computational cost could be established. The considered model is

\begin{tabular}{|c|c|c|}
\hline Model & Parameters vector & RSME \\
\hline$y_{1-\text { order }}(t)=\frac{a_{1 x} q^{-1}}{1-b_{1 x} q^{-1}} u(t)$ & $\begin{array}{c}\text { Repressors vector } \\
\phi(t)=\left[\begin{array}{ll}y(t-1) & u(t-1)\end{array}\right]\end{array}$ & 0.095 \\
\hline$y_{2-\text { order }}(t)=\frac{a_{1 x} q^{-1}+a_{2 x} q^{-2}}{1-b_{1 x} q^{-1}-b_{2 x} q^{-2}} u(t)$ & $\begin{array}{c}\text { Parameters vector } \\
\theta=\left[\begin{array}{ll}a_{1 x} & a_{2 x} \\
b_{1 x} & b_{2 x}\end{array}\right] \\
\text { Repressors vector } \\
\phi(t)=\left[\begin{array}{ll}y(t-1) & u(t-1) \\
y(t-2) & u(t-2)\end{array}\right]\end{array}$ & 0.0253 \\
\hline$y_{3-\text { order }}(t)=\frac{a_{1 x} q^{-1}+a_{2 x} q^{-2}+a_{3 x} q^{-3}}{1-b_{1 x} q^{-1}-b_{2 x} q^{-2}-b_{3 x} q^{-3}} u(t)$ & $\begin{array}{c}\text { Parameters vector } \\
\theta=\left[\begin{array}{lll}a_{1 x} & a_{2 x} & a_{3 x} \\
b_{1 x} & b_{2 x} & b_{3 x}\end{array}\right] \\
\text { Repressors vector } \\
\phi(t)=\left[\begin{array}{ll}y(t-1) & u(t-1) \\
y(t-2) & u(t-2) \\
y(t-3) & u(t-3)\end{array}\right]\end{array}$ & 0.0178 \\
\hline
\end{tabular}
represented as follows:

Table 2. The considered models and corresponding parameters and regression vectors. 


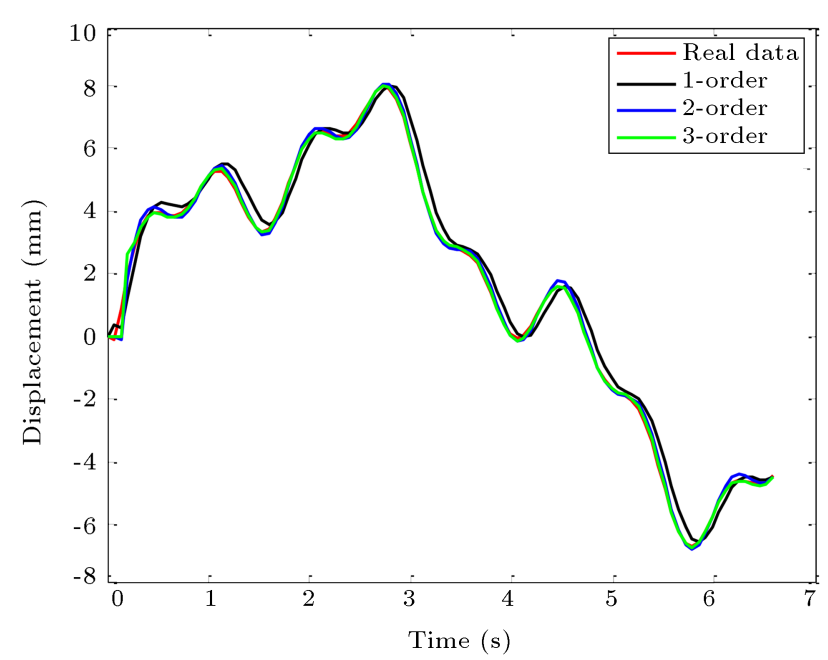

Figure 7. The comparison of the structures with different orders for predicting the system output.

$$
y_{A R X 2}(t)=\frac{a_{1 x} z^{-1}+a_{2 x} z^{-2}}{1+b_{1 x} z^{-1}+b_{2 x} z^{-2}} u(t) .
$$

After selecting the model order and defining it as a known parameter in the algorithm, the controller is designed and its performance under different operational conditions is investigated in the following section.

\subsection{Tuning the parameters of AGPC}

Tuning the AGPC parameters is of great significance considering its influence on the obtained responses. The parameters to be tuned are the lower and upper limits of the prediction horizon, the control horizon, sampling time, and weighting factor, $\lambda$. Several influential factors in determining these parameters should be considered. For example, to determine the prediction horizon, a slower response to the transient condition in the case of selecting the lower limit should be noted. Moreover, the control horizon implies the freedom degree of the controller and its value should not exceed the prediction horizon. Sampling time is also very important which affects the accuracy and computational cost. This value is mostly restricted due to hardware limitations. The weighting parameter, $\lambda$, affects the stability, performance, and accuracy of the results. Hence, regarding these points, the considered parameters for the predictive control are provided in Table 3.

Table 3. The Adaptive Generalized Predictive Control (AGPC) parameters.

\begin{tabular}{lc}
\hline \multicolumn{1}{c}{ AGPC parameter } & Value \\
\hline Prediction horizon-upper limit & 1 \\
Prediction horizon-lower limit & 5 \\
Control horizon & 4 \\
Sampling time & $0.001 \mathrm{~s}$ \\
$\lambda$ & 0.1 \\
\hline
\end{tabular}

\subsection{AGPC in normal conditions}

For evaluating the performance of the designed controller under normal conditions, the output of the system in response to varying step references is studied and the results are depicted in Figures 8 and 9 .

According to Figure 8, the output of the system tracks the desired reference after about 0.6 second, without steady state error. Moreover, upon changing the reference, approximately 0.2 second is required for the controller to track a new reference. Hence, the superior performance of the system in normal conditions (without considering any disturbance and uncertainty) can be concluded. The increment in the control effort as well as the control effort for following the desired reference are depicted in Figure 9.

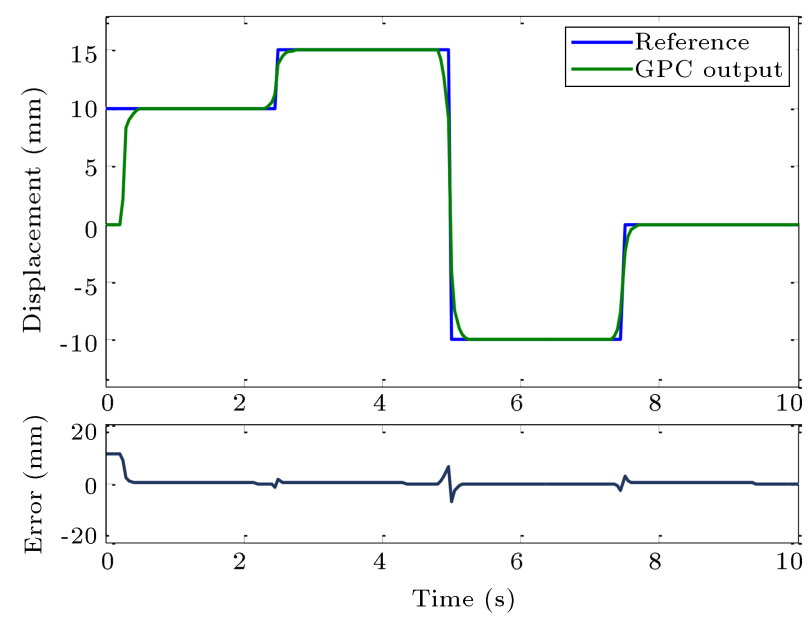

Figure 8. The performance of the proposed controller in the case of varying references (blue line is the reference and the green line represents the Generalized Predictive Control (GPC) output) in normal conditions along with the tracking error.
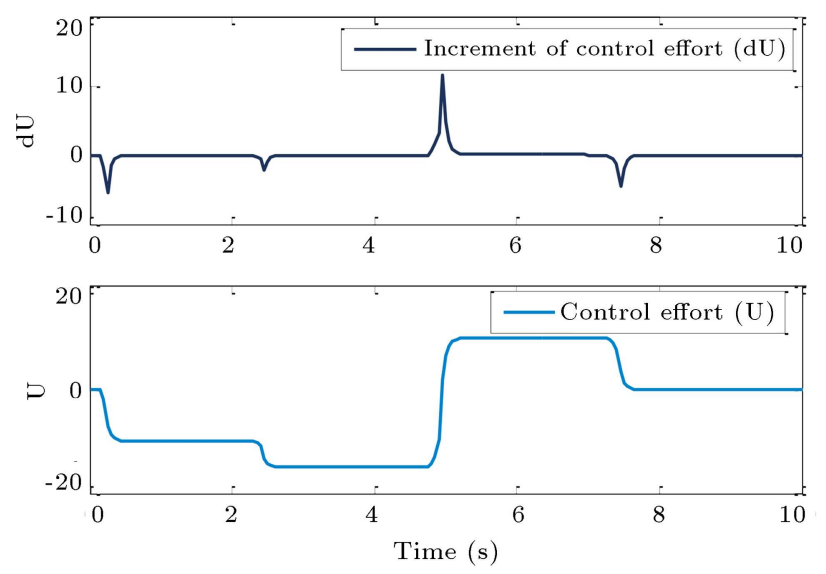

Figure 9. The increment of control effort and control effort of the proposed controller in the case of varying references (upper diagram represents the increment of the control effort and the lower diagram indicates the control effort) in normal conditions. 


\subsection{AGPC in the presence of disturbance}

The performance of the designed controller is also evaluated in the presence of external disturbances. For this purpose, a determined disturbance is applied to the system between 6 th and 7 th seconds as follows:

$$
\begin{aligned}
\text { dist }= & 0.9 \times[(t>6)(t<=6.5)] \\
& -0.6 \times[(t>6.5)(t<=7)] .
\end{aligned}
$$

The performance of the actuation system in the presence of this disturbance is depicted in Figures 10 and 11. According to the results depicted in Figure 10, the controller damps the disturbance completely after 0.5 second. Moreover, the increment in control effort and control effort for damping the effect of disturbance is presented in Figure 11.

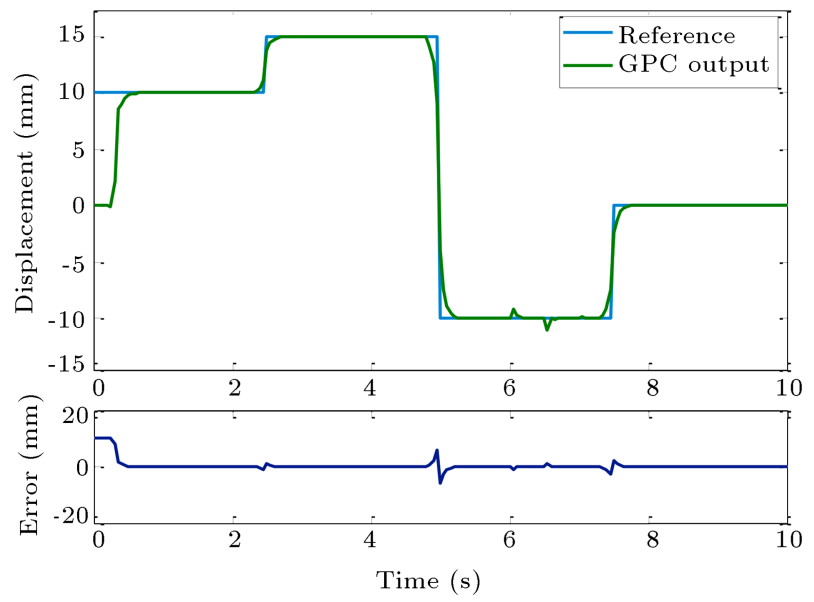

Figure 10. The performance of the proposed controller in the case of varying references (blue line is the reference and the green line represents the system output) in the presence of disturbance along with the tacking error.
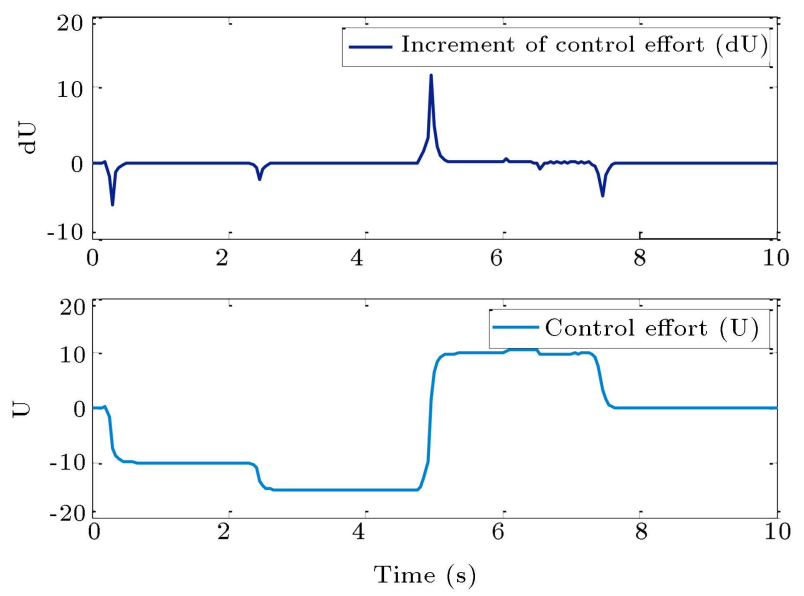

Figure 11. Increment in control effort and control effort of the proposed controller in the case of varying references (upper diagram represents the increment in the control effort and the lower diagram indicates the control effort) in the presence of disturbance.

\section{5. $A G P C$ in the presence of parametric uncertainties}

In this section, the performance of the system in the presence of parametric uncertainties is studied to evaluate the robustness of the proposed controller. To evaluate the effect of uncertainties on the controller performance, a set of tests at different levels of uncertainties in different parameters of the plant was conducted. The considered levels of uncertainties in the parameters of the system were $10 \%, 15 \%$, and $30 \%$ for parameters of the plant transfer function, $a_{1}, a_{2}, b_{1}$, and $b_{2}$. The corresponding results for $a_{1}, a_{2}, b_{1}$, and $b_{2}$ are provided in Figures $12,13,14$, and 15, respectively. Figure 16 represents simultaneous uncertainties associated with all the parameters.

According to the obtained results, the designed controller is capable of dealing with uncertainties in an acceptable manner. The most influential parameter in terms of uncertainty, as can be seen in these figures, is $b_{1}$ according to Figure 14. $b_{1}$ is the parameter that determines the dominant pole of the system. Then, $b_{2}$ is the second influential parameter. $a_{1}$ and $a_{2}$ have the least influence on the model behavior. The designed controller compensates the existing uncertainties in the first moments of performance. According to Figure 15 , up to $30 \%$ uncertainties in all parameters can be handled well using the adaptive controlling algorithm; however, the performance of the system was deteriorated with higher uncertainties in the parameters.

To ensure a better evaluation of the controller performance as well as comparison of the effects of un-

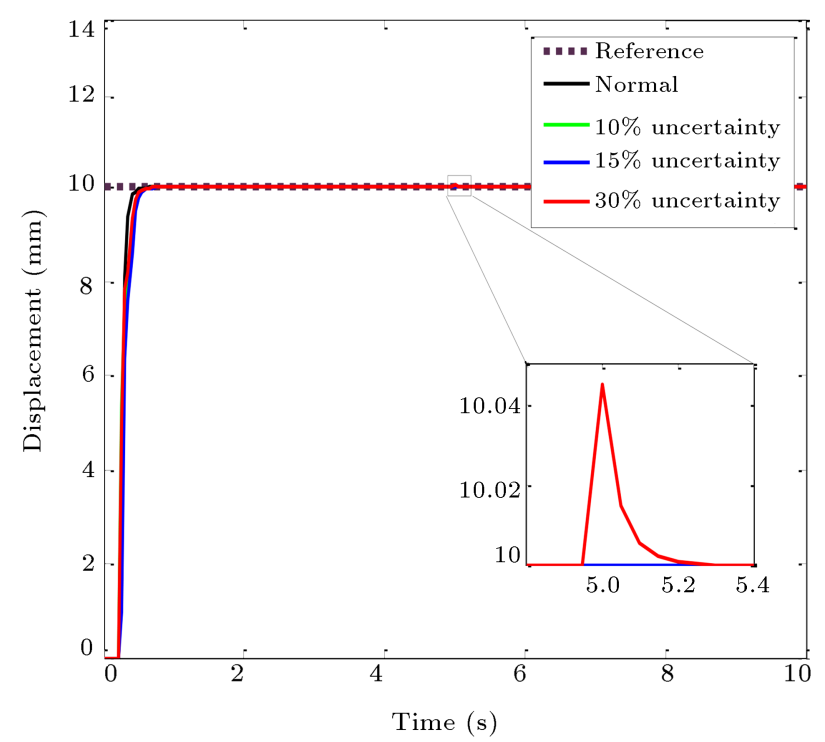

Figure 12. The performance of the proposed controller in the case of varying references and in the presence of uncertainties in $a_{1}$ parameter (dashed line is the reference, black line is normal condition, green line represents $10 \%$ uncertainty, blue line indicates $15 \%$ uncertainty, and red line shows $30 \%$ uncertainty). 


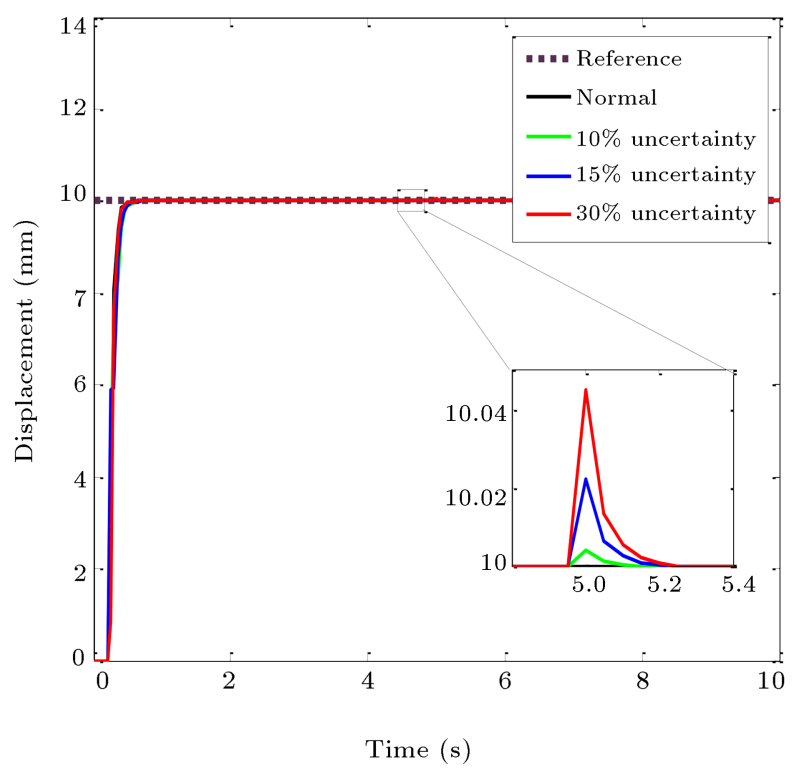

Figure 13. The performance of the proposed controller in the case of varying references and in the presence of uncertainties in $a_{2}$ parameter (dashed line is the reference, black line is normal condition, green line represents $10 \%$ uncertainty, blue line indicates $15 \%$ uncertainty, and red line shows $30 \%$ uncertainty).

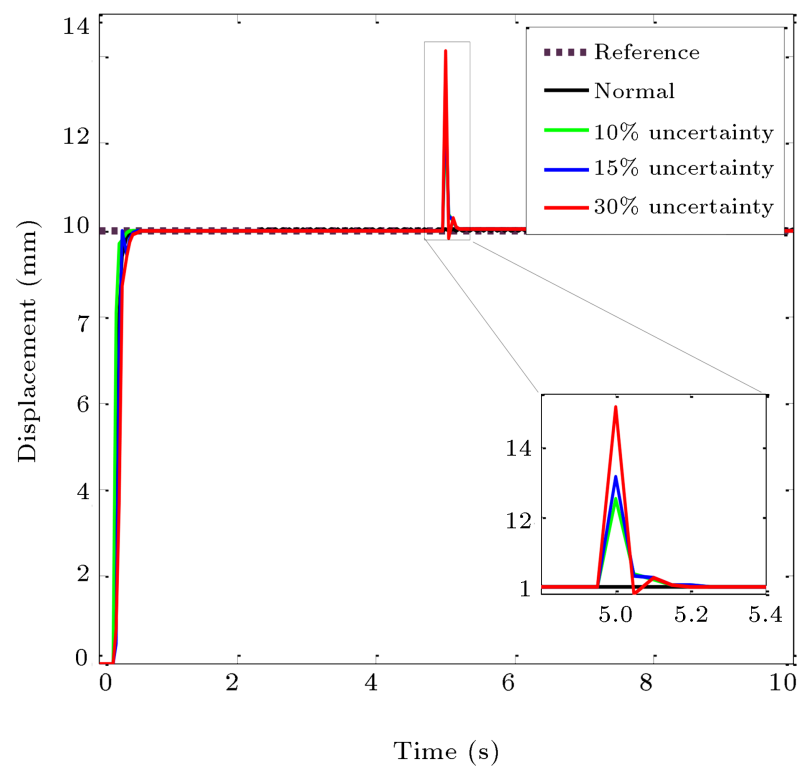

Figure 14. The performance of the proposed controller in the case of varying references and in the presence of uncertainties in $b_{1}$ parameter (dashed line is the reference, black line is normal condition, green line represents $10 \%$ uncertainty, blue line indicates $15 \%$ uncertainty, and red line shows $30 \%$ uncertainty).

certainty in different parameters, error-based indexes including RMSE, Mean Absolute Error (MAE), error mean, and Standard Deviation (Std) are evaluated. These criteria are defined in the following and the results are provided in Table 4.

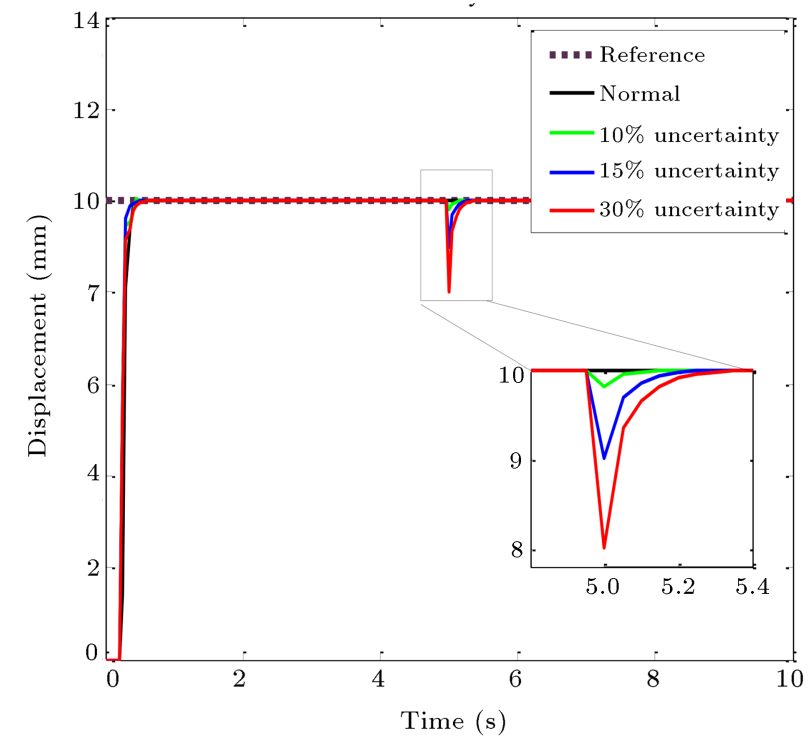

Figure 15. The performance of the proposed controller in the case of varying reference and in the presence of uncertainties in $b_{2}$ parameter (dashed line is the reference, black line is normal condition, green line represents $10 \%$ uncertainty, blue line indicates $15 \%$ uncertainty, and red line shows $30 \%$ uncertainty).

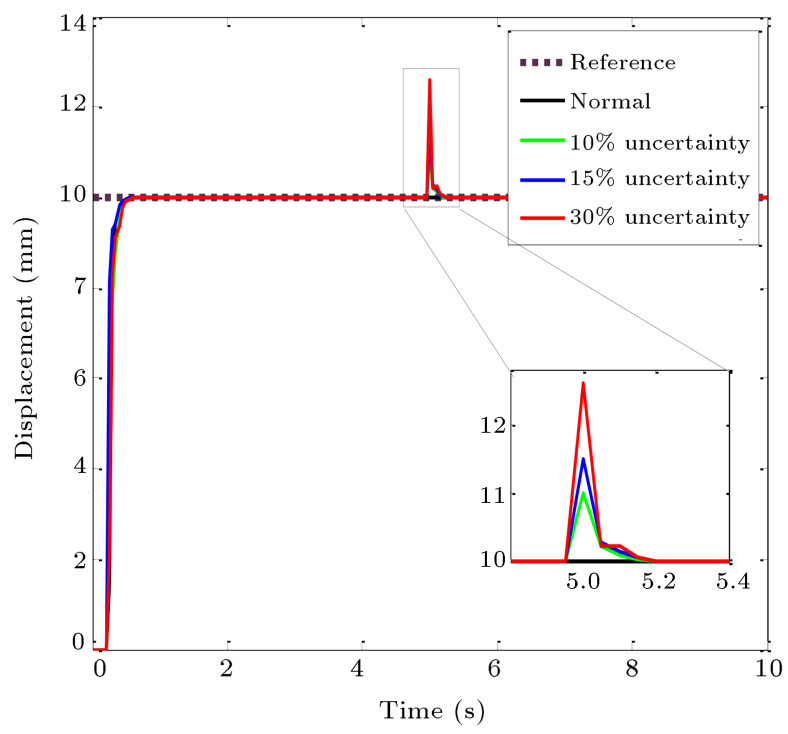

Figure 16. The performance of the proposed controller in the case of varying references and in the presence of uncertainties in all parameters (dashed line is the reference, black line is normal condition, green line represents $10 \%$ uncertainty, blue line indicates $15 \%$ uncertainty, and red line shows $30 \%$ uncertainty).

$$
\begin{aligned}
& R M S E=\sqrt{\left(\sum_{k=1}^{n_{\text {data }}}\left\|(y(k)-\hat{y}(k))^{2}\right\|_{2}\right) / n_{d},} \\
& M A E=\frac{1}{n_{\text {data }}} \sum_{k=1}^{n_{\text {data }}}|y(k)-\hat{y}(k)|,
\end{aligned}
$$


Table 4. The performance comparison in terms of the uncertainties in different parameters.

\begin{tabular}{|c|c|c|c|c|c|}
\hline \multirow[b]{2}{*}{ Parameters } & \multirow[b]{2}{*}{ Index } & \multicolumn{4}{|c|}{ Uncertainty } \\
\hline & & $\begin{array}{c}0 \% \\
\text { (normal) }\end{array}$ & $10 \%$ & $15 \%$ & $30 \%$ \\
\hline \multirow{4}{*}{$a_{1}$} & RMSE & 0.1578 & 0.1583 & 0.1606 & 0.1663 \\
\hline & Mean & 0.2510 & 0.2598 & 0.2701 & 0.2827 \\
\hline & Std & 0.1554 & 0.1565 & 0.1587 & 0.1640 \\
\hline & MAE & 0.2610 & 0.2640 & 0.2720 & 0.3130 \\
\hline \multirow{4}{*}{$a_{2}$} & RMSE & 0.1578 & 0.1617 & 0.1631 & 0.17129 \\
\hline & Mean & 0.2510 & 0.2793 & 0.2998 & 0.0310 \\
\hline & Std & 0.1554 & 0.1591 & 0.1608 & 0.1688 \\
\hline & MAE & 0.2610 & 0.2790 & 0.3001 & 0.312 \\
\hline \multirow{4}{*}{$b_{1}$} & RMSE & 0.1578 & 0.1694 & 0.1767 & 0.1803 \\
\hline & Mean & 0.2510 & 0.2808 & 0.0300 & 0.0311 \\
\hline & Std & 0.1554 & 0.1679 & 0.1746 & 0.1781 \\
\hline & MAE & 0.2610 & 0.2890 & 0.3380 & 0.0366 \\
\hline \multirow{4}{*}{$b_{2}$} & RMSE & 0.1578 & 0.1607 & 0.1618 & 0.1715 \\
\hline & Mean & 0.2510 & 0.0270 & 0.0278 & 0.2924 \\
\hline & Std & 0.1554 & 0.1588 & 0.1588 & 0.1592 \\
\hline & MAE & 0.2610 & 0.2780 & 0.2810 & 0.2930 \\
\hline
\end{tabular}

$$
\begin{aligned}
& E_{\text {mean }}=\frac{1}{n_{\text {data }}} \sum_{k=1}^{n_{\text {data }}}(y(k)-\hat{y}(k)), \\
& S t d=\frac{1}{n_{\text {data }}} \sqrt{\sum\left(y(k)-E_{\text {mean }}\right)^{2}} .
\end{aligned}
$$

In the above formulations, $y(k)$ and $\hat{y}(k)$ are the real and estimated values of the output. In addition, $n_{d a t a}$ is data size. According to Table 4, as mentioned before, $b_{1}$ is the most influential parameter in uncertainty investigation. The increase in RMSE, mean, Std, and MAE of this parameter is equal to $14.1,21,14.8$, and $24.1 \%$ for $30 \%$ uncertainty compared to the normal condition.

\section{Conclusion}

In this paper, an adaptive case of Model Predictive Control (MPC) algorithm known as Adaptive Generalized Predictive Control (AGPC) was used for controlling the actuation system of high-powered test rigs for gearboxes. The model of the system was obtained through online system identification according the data gathered from the experimental tests. Precise control of the actuation system is of great importance considering the influence on the health evaluation of the gearbox. Through efficient control of the actuation system, provision of different conditions becomes possible. Furthermore, in order to reduce the computational cost, two schemes were utilized: (a) parametric identification with known order and (b) parametric calculation of the control sequence instead of calculating the control increment over the whole horizon. Different conditions were considered to evaluate the performance of the proposed control scheme including the presence of external disturbance and parametric uncertainties. According to the obtained results, in normal conditions, the controller output tracked the reference in $0.6 \mathrm{~s}$ without steady state error. Moreover, approximately 0.2 second was required for the controller after changing the reference for tracking. In case of the presence of the determined disturbance, the controller could damp the disturbance after $0.1 \mathrm{~s}$ completely. Furthermore, the performance of the controller in the presence of different magnitudes of uncertainties $(10 \%, 25 \%$, and $30 \%$ ) for different parameters of the transfer functions $\left(a_{1}, a_{2}, b_{1}\right.$, and $b_{2}$ ) was also investigated. As demonstrated by the results, the effect of parameter was the most influential parameter among the studied parameters. However, the system can overcome the uncertainty even in case of $30 \%$ uncertainty. Hence, the adaptability and robustness for the performance of the proposed control scheme were confirmed.

\section{Nomenclature}

$\begin{array}{ll}A, B, C & \text { System polynomials } \\ E(j), F(j) & \text { Polynomials of Diophantine equation } \\ J & \text { Cost function } \\ S_{e x c} & \text { Excitation signal } \\ \mathrm{TF} & \text { Transfer Function } \\ W_{\text {des }} & \text { Desired reference } \\ k & \text { Discrete time sample } \\ l & \text { Number of sine signal } \\ n & \text { System order } \\ n a, n b, n c & \text { Degrees of polynomials } A, B, C \\ P & \text { Prediction horizon } \\ S_{i} & \text { Amplitude of excitation signals } \\ t_{s} & \text { Sampling time } \\ u(k) & \text { System input } \\ V & \text { Control horizon } \\ y(k) & \text { System output } \\ Z Z^{-1} & \text { One-step backward operator } \\ \omega_{i} & \text { Frequency of excitation signals } \\ \phi(t) & \text { Regression vector } \\ \lambda(j) & \text { Control weighting coefficient } \\ \xi(k) & \text { Uncorrelated random noise with zero } \\ & \text { mean }\end{array}$




\section{References}

1. Zhou, L., Duan, F., Corsar, M., et al. "A study on helicopter main gearbox planetary bearing fault diagnosis", Appl. Acoust., 147, pp. 4-14 (2019).

2. Defreyne, P., Dereyne, S., Stockman, K., et al. "An energy efficiency measurement test bench for gearboxes", EEMODS, 2013, pp. 1-12 (2013).

3. Tri, N.M., Nam, D.N.C., Park, H.G., et al. "Trajectory control of an electro hydraulic actuator using an iterative backstepping control scheme", Mechatronics, 29, pp. 96-102 (2015).

4. Lee, W. and Chung, W.K. "Disturbance-observerbased compliance control of electro-hydraulic actuators with backdrivability", IEEE Robot. Autom. Lett., 4(2), pp. 1722-1729 (2019).

5. Huang, J., An, H., Yang, Y., et al. "Model predictive trajectory tracking control of electro-hydraulic actuator in legged robot with multi-scale online estimator", IEEE Access, 8, pp. 95918-95933 (2020).

6. Mohammed, S., Soon, C.C., Ghazali, R., et al. "An electro-hydraulic servo with intelligent control strategy", In MATEC Web of Conferences, 150, p. 1016 (2018).

7. Lu, X., Bai, Y., Fan, B., et al. "A hybrid offline/online modeling based tracking control for complex hydraulic driving processes", IEEE Access, 7, pp. 106102-106110 (2019).

8. Palermo, A., Toso, A., Heirman, G.H.K., et al. "Structural coupling and non-linear effects in the experimental modal analysis of a precision gear test rig", In International Gear Conference, 2014, pp. 1049-1059 (2014).

9. Mihailidis, A. and Nerantzis, I. "A new system for testing gears under variable torque and speed", Recent Patents Mech. Eng., 2(3), pp. 179-192 (2009).

10. Huang, J., An, H., Lang, L., et al. "A data-driven multi-scale online joint estimation of states and parameters for electro-hydraulic actuator in legged robot", IEEE Access, 8, pp. 36885-36902 (2020).

11. Yao, Z., Yao, J., and Sun, W. "Adaptive RISE control of hydraulic systems with multilayer neural-networks", IEEE Trans. Ind. Electron., 66(11), pp. 8638-8647 (2018).

12. Takloo, S.D., Mozafari, S., Rezazadehmohamadi, M., et al. "Fractional order PID control mechanism for helicopter gearbox test control with internal and external disturbance", Bull. la Société R. des Sci. Liége, 86, special issue, pp. 127-138 (2017).
13. Maddahi, A., Sepehri, N., and Kinsner, W. "Fractional -order control of hydraulically powered actuators: Controller design and experimental validation", IEEE/ASME Trans. Mechatronics, 24(2), pp. 796-807 (2019).

14. Li, M., Shi, W., Wei, J., et al. "Parallel velocity control of an electro-hydraulic actuator with dual disturbance observers", IEEE Access, 7, pp. 56631-56641 (2019).

15. Heybroek, K. and Sjjberg, J. "Model predictive control of a hydraulic multichamber actuator: A feasibility study", IEEE/ASME Trans. Mechatronics, 23(3), pp. 1393-1403 (2018).

16. Rozali, S.M., Rahmat, M.F., Wahab, N.A., et al. "PID controller design for an industrial hydraulic actuator with servo system", In 2010 IEEE Student Conference on Research and Development (SCOReD), pp. 218-223 (2018).

17. Pan, Y. and Yu, H. "Composite learning from adaptive dynamic surface control", IEEE Trans. Automat. Contr., 61(9), pp. 2603-2609 (2015).

18. Yang, J., Na, J., and Gao, G. "Robust model reference adaptive control for transient performance enhancement", Int. J. Robust Nonlinear Control, 30(15), pp. 6207-6228 (2020).

19. Yang, J., Na, J., and Gao, G. "Robust adaptive control for unmatched systems with guaranteed parameter estimation convergence", Int. J. Adapt. Control Signal Process., 33(12), pp. 1868-1884 (2019).

20. Camacho, E.F. and Alba, C.B. "Model predictive control" In Springer Science \& Business Media (2013).

21. Balaji, V. and Maheswari, E. "Model predictive control strategy for industrial process", Bull. Electr. Eng. Informatics, 1(3), pp. 191-198 (2012).

22. Alamirew, T., Balaji, V., and Gabbeye, N. "Comparison of PID controller with model predictive controller for milk pasteurization process", Bull. Electr. Eng. Informatics, 6(1), pp. 24-35 (2017).

23. Parvaresh, A. and Mardani, M. "Model predictive control of a hydraulic actuator in torque applying system of a mechanically closed-loop test rig for the helicopter gearbox", Aviation, 23(4), pp. 143-153 (2019).

24. Wang, D., Zhao, D., Gong, M., et al. "Research on robust model predictive control for electro-hydraulic servo active suspension systems", IEEE Access, 6, pp. 3231-3240 (2017).

25. Parvaresh, A. and Mardani, M. "Data-driven modelfree control of torque-applying system for a mechanically closed-loop test rig using neural networks", Stroj. Vestnik/Journal Mech. Eng., 66(5), (2020).

26. Raziei, S.A. and Jiang, Z. "Dynamic modeling and nonlinear model predictive control of hybrid actuator systems", In 2017 IEEE National Aerospace and Electronics Conference (NAECON), pp. 119-126 (2017). 
27. Pereida, K. and Schoellig, A.P. "Adaptive model predictive control for high-accuracy trajectory tracking in changing conditions", In 2018 IEEE/RSJ International Conference on Intelligent Robots and Systems (IROS), pp. 7831-7837 (2018).

28. He, W.J., Zhang, H.T., Chen, Z., et al. "Generalized predictive control of temperature on an atomic layer deposition reactor", IEEE Trans. Control Syst. Technol., 23(6), pp. 2408-2415 (2015).

29. Gao, Z. "On the centrality of disturbance rejection in automatic control", ISA Trans., 53(4), pp. 850-857 (2014).

30. Kim, J.S. "Recent advances in adaptive MPC", In ICCAS 2010, pp. 218-222 (2010).

31. Heirung, T.A.N., Ydstie, B.E., and Foss, B. "Dual adaptive model predictive control", Automatica, 80, pp. $340-348$ (2017).

32. Dong, N., Feng, Y., Han, X.S., et al. "An improved model-free adaptive predictive control algorithm for nonlinear systems with large time delay", In 2018 IEEE 7th Data Driven Control and Learning Systems Conference (DDCLS), pp. 60-64 (2018).

33. Guo, Y., Hou, Z., Liu, S., et al. "Data-driven modelfree adaptive predictive control for a class of MIMO nonlinear discrete-time systems with stability analysis", IEEE Access, 7, pp. 102852-102866 (2019).

34. Wang, Z. and Wang, J. "Ultra-local model predictive control: A model-free approach and its application on automated vehicle trajectory tracking", Control Eng. Pract., 101, p. 104482 (2020).

35. Clarke, D.W. "Application of generalized predictive control to industrial processes", IEEE Control Syst. Mag., 8(2), pp. 49-55 (1988).
36. Hyatt, P. and Killpack, M.D. "Real-time evolutionary model predictive control using a graphics processing unit", In 2017 IEEE-RAS 17th International Conference on Humanoid Robotics (Humanoids), pp. 569-576 (2017).

37. Söderström, T. and Stoica, P. "System identification", In Prentice-Hall International (1989).

38. Ishak, N., Tajjudin, M., Ismail, H., et al. "System identification and model validation of electro-hydraulic actuator for quarter car system", WSEAS Trans. Adv. Eng. Educ, 4, pp. 27-35 (2017).

39. Ljung, L., System Identification, Wiley Encycl. Electr. Electron. Eng., pp. 100-129 (1999).

\section{Biographies}

Aida Parvaresh received her BS degree in 2011 and MS degree in 2013 in Mechanical Engineering from Tabriz University. She is currently a $\mathrm{PhD}$ candidate at the Mechanical Engineering Department of K.N. Toosi University of Technology. Her research interest is in the areas of dynamics modeling, system identification, and control of robotics and industrial systems.

Mohsen Mardani received his BS degree of Electrical Engineering in 2005. He also received MSc and PhD degrees in Energy Engineering from Amirkabir University of Technology in 2008 and 2013, respectively. He also worked as a Research Assistant at Nanyang Technological University, Singapore in 2013. Currently, he is an Associate Professor in Sharif Technology branch of ACECR and his current position is the Vice President of Research and Technology. His research interests include test systems and their applications. 